\title{
Absence of Autophagy-Related Proteins Expression Is Associated with Poor Prognosis in Patients with Colorectal Adenocarcinoma
}

\author{
Ji Hye Choi, ${ }^{1}$ Young-Seok Cho, ${ }^{2}$ Yoon Ho Ko, ${ }^{2}$ Soon Uk Hong, ${ }^{3}$ \\ Jin Hee Park, ${ }^{1}$ and Myung Ah Lee ${ }^{4}$ \\ ${ }^{1}$ Department of Biomedical Science, The Catholic University of Korea College of Medicine, Seoul 137-701, Republic of Korea \\ ${ }^{2}$ Department of Internal Medicine, Uijeongbu St. Mary's Hospital, The Catholic University of Korea College of Medicine, \\ Uijeongbu 480-717, Republic of Korea \\ ${ }^{3}$ Department of Pathology, Asan Medical Center, University of Ulsan College of Medicine, Seoul 138-736, Republic of Korea \\ ${ }^{4}$ Department of Internal Medicine, Seoul St. Mary's Hospital, The Catholic University of Korea College of Medicine, \\ Seoul 137-701, Republic of Korea
}

Correspondence should be addressed to Young-Seok Cho; yscho@catholic.ac.kr and Yoon Ho Ko; koyoonho@catholic.ac.kr

Received 17 December 2013; Accepted 21 January 2014; Published 5 March 2014

Academic Editor: Robert Odze

Copyright (C) 2014 Ji Hye Choi et al. This is an open access article distributed under the Creative Commons Attribution License, which permits unrestricted use, distribution, and reproduction in any medium, provided the original work is properly cited.

\begin{abstract}
Background/Aim. Autophagy, a cellular degradation process, has paradoxical roles in tumorigenesis and the progression of human cancers. The aim of this study was to investigate the expression levels of autophagy-related proteins in colorectal cancer (CRC) and to evaluate their prognostic significance. Methods. This study is a retrospective review of immunohistochemical and clinicopathological data. All specimens evaluated were obtained from 263 patients with colorectal cancer who had undergone surgery between November 1996 and August 2007. The primary outcomes measured were the expression levels of three autophagy-related proteins (ATG5, BECN1/Beclin 1, and Microtubule-associated protein 1 light chain 3B (LC3B)) by immunohistochemistry and its association in clinicopathological parameters and patient survival. Results. The autophagy-related protein expression frequencies were $65.1 \%$ (151/232) for ATG5, 71.3\% (174/244) for BECN1, and 74.7\% (186/249) for LC3B for the 263 patients. Correlation between the expression of autophagy-related proteins was significant for all protein pairs. Multivariate analysis showed that negative LC3B expression and absence of autophagy-related proteins expression were independently associated with poor prognosis. Conclusion. Absence of autophagy-related proteins expression is associated with poor clinical outcome in CRC, suggesting that these proteins have potential uses as novel prognostic markers.
\end{abstract}

\section{Introduction}

Autophagy is an evolutionarily conserved catabolic process in which the cell self-digests excessive, damaged, or aged proteins and damaged organelles [1]. This cellular process is characterized by the formation of autophagosomes, doublemembraned vesicles that sequester the cytoplasmic material that is destined for degradation in the lysosome [2]. Autophagy occurs at low basal levels in most cells to maintain cellular homeostasis. However, this process is upregulated in response to metabolic stresses, including starvation, hypoxia, and growth factor deprivation, to generate intracellular nutrients and energy [1]. Autophagy is involved in the regulation of many physiological and pathological processes, including cell development and differentiation, immunity, energy homeostasis, cell death, and tumorigenesis [2]. The role of autophagy in tumorigenesis is complex and paradoxical [3]. Autophagy defects can accelerate tumorigenesis. The mammalian autophagy gene $B E C N 1 / B e c l i n ~ 1$ is frequently monoallelically deleted in many cancers, including human ovarian, breast, and prostate cancers, and mice with allelic loss of BECN1 are more likely to develop hepatocellular carcinoma (HCC), lung adenocarcinomas, lymphomas, and mammary hyperplasia $[4,5]$. However, other studies have suggested that the prosurvival function of autophagy under stress conditions can promote tumor development [6-8]. 
In addition, the pharmacological or genetic inhibition of autophagy has been shown to enhance the cytotoxic effects of chemotherapeutic agents [9-11].

Colorectal cancer (CRC) is one of the leading causes of cancer-related mortality worldwide [12]. Although the potential of several molecular markers, including the TP53 or KRAS mutational status and loss of heterozygosity in chromosome $18 \mathrm{q}$, has been investigated to identify patients with a greater likelihood of recurrence after curative resection, the results are conflicting [13-15]. A previous study demonstrated that autophagy is activated in CRC in vitro and in vivo, and autophagy may contribute to the survival of colorectal cancer cells that have acquired resistance to nutrient starvation [16].

Autophagy-related genes (ATGs) regulate and implement the autophagy process, and more than 30 ATGs have been identified in yeast, of which 20 have also been found in humans [17]. Among these genes, BECN1 (a mammalian homolog of the yeast ATG6 protein) is an essential modifier of the autophagic process and has been implicated in the tumorigenesis of many types of cancers [18]. Microtubuleassociated protein 1 light chain 3 (LC3) is an autophagosomal ortholog of the yeast ATG8 and exists in two forms, LC3-I and its proteolytic derivative LC3-II. The activation of autophagy in response to various stresses stimulates the conversion of LC3-I into LC3-II and upregulates LC3 expression. LC3 is a specific marker of autophagosome formation and is the most widely monitored autophagy-related protein [19]. ATG5 is a key regulatory protein involved in the early stage of autophagosome formation. The conjugation of ATG5 with ATG12 through an ubiquitin-like system contributes to autophagosome formation [20]. In the present study, we evaluated the expression of autophagy-related proteins (ATG5, BECN1, and LC3) in primary colorectal adenocarcinomas and their relationship with clinicopathological parameters and clinical outcomes.

\section{Materials and Methods}

2.1. Patients and Tumor Samples. All specimens evaluated in the present study were obtained from patients with CRC between November 1996 and August 2007 at Seoul St. Mary's Hospital of the Catholic University of Korea. During the study period, a total of 298 consecutive patients with CRC who had undergone surgery were enrolled. Patients were included in the present study if the survival time was known and paraffin blocks of tumor specimens were available. Patients were excluded from this study due to surgery-related mortality $(n=10)$, poor quality of paraffin-block $(n=$ 15 ), and loss of tissue during the construction of tissue microarray blocks $(n=10)$. Therefore, 263 pathologically confirmed specimens were investigated. None of the patients had received either radiotherapy or chemotherapy preoperatively. We reviewed the medical records of the patients and obtained clinicopathological data, including age, sex, histopathological diagnosis, and pathological tumor stage, and patient outcomes, such as last follow-up and recurrence. Cancer-related death was identified based on medical records and/or telephone interviews. The World Health Organization criteria were used for histological classification, and the TNM classification system was used for postoperative pathological staging according to the 7th edition of the American Joint Committee on Cancer staging criteria. This study was approved by the Institutional Research Ethics Board of Seoul St. Mary's Hospital of the Catholic University of Korea.

2.2. Tissue Microarray Methods. Tissue microarray recipient blocks were constructed from archival formalin-fixed, paraffin-embedded tissue blocks prepared from primary colorectal cancer specimens according to established methods [21]. Briefly, morphologically representative tissue areas marked on standard hematoxylin and eosin-stained sections were punched from donor blocks, and the tissue cores were placed in the recipient blocks. In total, 30 cores were assembled in a recipient block. After construction, $4 \mu \mathrm{m}$ sections were cut from the array block and transferred to glass slides.

2.3. Immunohistochemistry and Analysis. Using sections from the tissue microarrays, immunohistochemistry was performed using a Lab Vision Autostainer LV-1 (LabVision/ Neomarkers, Fremont, CA) according to the manufacturer's protocol. The primary antibodies were rabbit polyclonal antibodies against ATG5, BECN1, and LC3B purchased from Abcam (Cambridge, UK). They were used at the following dilutions: ATG5 (1:2000), BECN1 $(1: 130)$, and LC3B $(1: 200)$. The samples were incubated with the primary antibodies at room temperature for 24 hours, and immunoreactivity was detected by a conventional streptavidin-biotin labeling method (LSAB2 System; Dako, Carpinteria, CA). For the negative controls, sections were treated using the same method with the exception that they were incubated with the antibody diluents instead of the primary antibodies. Immunostaining patterns were interpreted using a semiquantitative histological score according to our previous study [22]. The intensity of immunoreactivity was classified as negative $(0)$, weak $(1+)$, moderate $(2+)$, or strong $(3+)$. The percent area occupied by immunoreactive tumor cells was classified as grade $0(0 \%), 1(1 \%-30 \%)$, or $2(31 \%-100 \%)$. A composite score was calculated by multiplying the intensity and percentage scores. In the evaluation of autophagy-related protein expression, a composite score of 0 was considered negative protein expression, and scores of 1 to 3 were defined as positive protein expression.

2.4. Statistical Analysis. Statistical analyses were performed using the SPSS software package (version 13.0; SPSS, Chicago, IL). The Chi-square test, Fisher's exact test, and JonckheereTerpstra test were used for the analysis of the relationship between the immunohistochemical profiles and the clinicopathological variables. The "time-to-event" data were evaluated by the Kaplan-Meier method, and significant differences between the groups were identified by the log-rank test. The Cox proportional hazards regression model was used to identify the factors related to overall survival (OS). All variables with a $P<0.2$ in the univariate analysis were included in the multivariate analysis. Overall survival (OS) was calculated from the date of diagnosis to the date of death or the last follow-up. Survival rates and odds ratios are presented with their respective $95 \%$ confidence intervals 
(CIs). A value of $P$ of $<0.05$ was considered statistically significant.

\section{Results}

3.1. Patients' Clinical Characteristics. In total, 263 paraffin blocks of tumor samples were available from patients who had undergone surgery. Table 1 shows the clinicopathological characteristics of the patients. The patient cohort consisted of 141 males and 122 females, with a median age of 64 years (range, 30-83 years). Histologically, most patients (88.6\%) had tubular adenocarcinoma. According to the American Joint Committee on Cancer staging criteria, 38 patients (14.4\%) had stage I disease, 67 (25.5\%) had stage II disease, $101(38.4 \%)$ had stage III disease, and 57 (21.7\%) had stage IV disease. All patients with stage IV received palliative resection for primary CRC. Tissue microarrays were constructed with samples obtained from primary CRC, not metastatic sites. One hundred and twenty (45.6\%) patients underwent adjuvant chemotherapy and/or radiation therapy, 74 (28.1\%) received 5-fluorouracil (FU)-based chemotherapy, and 46 $(17.5 \%)$ received 5-FU-based concurrent chemoradiation or radiation alone. The median follow-up duration was 71.4 months (range, 0.5-197.4 months) after surgical resection. Among the 263 patients, 87 (33.1\%) died of their tumors and 176 (66.9\%) were alive at the last follow-up.

3.2. Expression of Autophagy-Related Proteins. Figure 1 shows representative immunohistochemistry results. The autophagy-related protein expression frequencies were $65.1 \%$ (151/232) for ATG5, 71.3\% (174/244) for BECN1, and 74.7\% (186/249) for LC3B among the 263 patients (Figure 2). The expression of autophagy-related proteins in tumor cells was predominantly localized to the cytoplasm. The associations between the expression of autophagy-related proteins and clinicopathological parameters, including well-known prognostic factors such as histological differentiation, lymphovascular invasion and lymph node metastasis, were investigated (Table 2). Positive BECN1 expression was significantly correlated with good histological differentiation $(P=0.035)$. However, no significant correlation was observed between the expression levels of autophagy-related proteins and the clinicopathological parameters. In addition, the relationships between the expression patterns of different autophagyrelated proteins were assessed (Table 3 ). The correlations between the expression patterns of autophagy-related proteins were statistically significant for all protein pairs.

3.3. Survival Analysis with respect to Clinicopathological Factors and Autophagy-Related Protein Expression. The overall 5 -year survival rate for resected CRCs was 79.5\%. The median overall survival time had not yet been reached for all patients. Univariate analysis of the clinicopathological parameters relevant to patient survival showed that the following factors were significantly associated with the overall survival of the patients: histological type $(P=0.004)$, TNM stage $(P<$ $0.001)$, lymphovascular invasion status $(P<0.001)$, the depth of invasion $(P<0.001)$, the degree of histological differentiation $(P=0.001)$, and curative resection status
TABLE 1: Baseline clinicopathological characteristics of patients with colorectal cancer.

\begin{tabular}{|c|c|c|}
\hline \multirow{2}{*}{ Characteristics } & \multicolumn{2}{|l|}{ Total } \\
\hline & Patient number & $\%$ \\
\hline Patient number & 263 & \\
\hline Age (years), median (range) & $64(30-83)$ & \\
\hline$<65$ & 141 & 53.6 \\
\hline$\geq 65$ & 122 & 46.4 \\
\hline \multicolumn{3}{|l|}{ Gender } \\
\hline Male & 141 & 53.6 \\
\hline Female & 122 & 46.4 \\
\hline \multicolumn{3}{|l|}{ Histology } \\
\hline Tubular adenocarcinoma & 233 & 88.6 \\
\hline Others* & 30 & 11.4 \\
\hline \multicolumn{3}{|l|}{ T stage } \\
\hline 1 & 1 & 0.4 \\
\hline 2 & 51 & 19.4 \\
\hline 3 & 163 & 62.0 \\
\hline 4 & 48 & 18.2 \\
\hline \multicolumn{3}{|l|}{$\mathrm{N}$ stage } \\
\hline 0 & 114 & 43.3 \\
\hline 1 & 80 & 30.4 \\
\hline 2 & 69 & 26.2 \\
\hline \multicolumn{3}{|l|}{ Stage } \\
\hline I & 38 & 14.4 \\
\hline II & 67 & 25.5 \\
\hline III & 101 & 38.4 \\
\hline IV & 57 & 21.7 \\
\hline \multicolumn{3}{|l|}{ Histological grade } \\
\hline Well/moderately & 222 & 84.4 \\
\hline Poorly & 41 & 15.6 \\
\hline \multicolumn{3}{|l|}{ Lymphovascular invasion } \\
\hline Negative & 99 & 37.6 \\
\hline Positive & 164 & 62.4 \\
\hline \multicolumn{3}{|l|}{ Site of primary tumor } \\
\hline Right colon & 69 & 26.2 \\
\hline Left colon & 83 & 31.6 \\
\hline Rectum & 111 & 42.2 \\
\hline \multicolumn{3}{|l|}{ Curative resection ( $\mathrm{R} 0$ resection) } \\
\hline Yes & 208 & 79.1 \\
\hline No & 55 & 20.9 \\
\hline \multicolumn{3}{|l|}{ Adjuvant therapy $(n=120)$} \\
\hline 5-FU-based chemotherapy & 74 & 28.1 \\
\hline 5-FU-based CCRT & 46 & 17.5 \\
\hline
\end{tabular}

${ }^{*}$ Mucinous carcinoma $(n=28)$, signet-ring-cell carcinoma $(n=2)$.

Data are numbers of patients with percentages in parentheses unless otherwise specified.

SD: standard deviation; T1: tumor invades submucosa; T2: tumor invades muscularis propria; T3: tumor invades through the muscularis propria into the subserosa or into the nonperitonealized pericolic or perirectal tissues; T4: tumor perforates the visceral peritoneum or directly invades other organs; N1: metastasis in 1-3 pericolic or perirectal lymph nodes; N2: metastasis in $>3$ pericolic or perirectal lymph nodes; 5-FU: 5-fluorouracil, CCRT: concurrent chemoradiation therapy. 


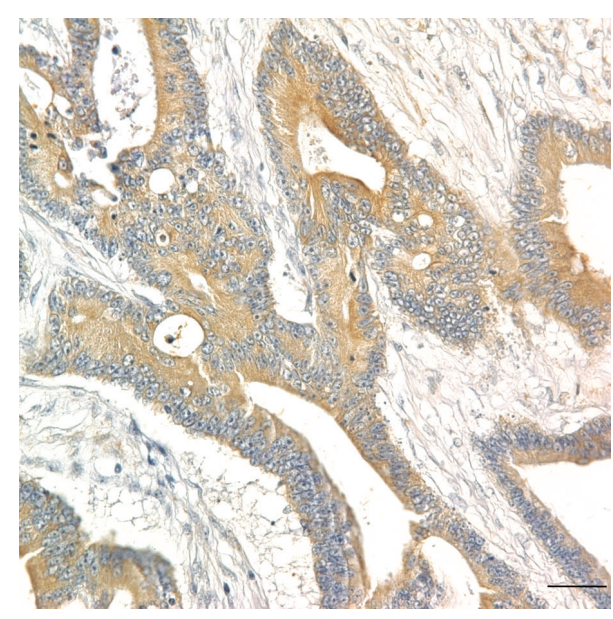

(a)

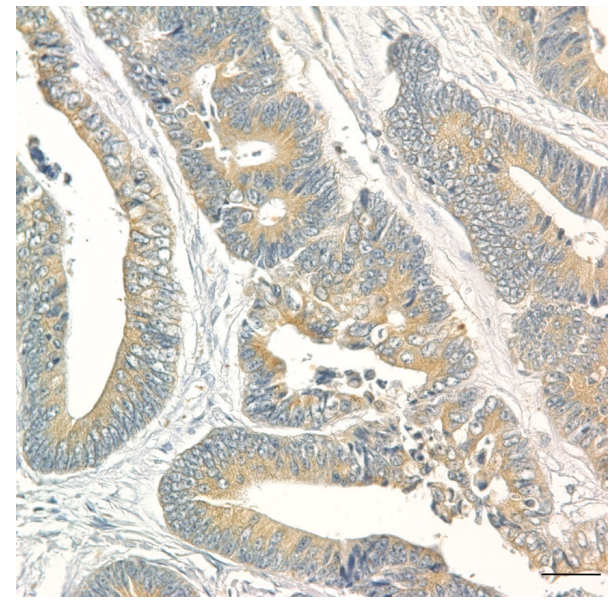

(b)

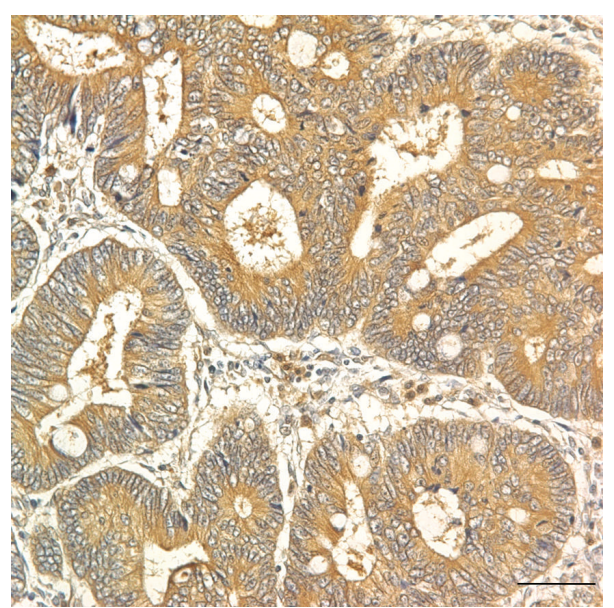

(c)

FIGURE 1: Immunohistochemistry findings show autophagy-related protein overexpression in CRC. Tumor cells show strong positive staining for ATG5 (a), BECN1 (b), and LC3B (c) proteins. Magnification, $\times 400$. Scale bars, $50 \mu \mathrm{m}$.

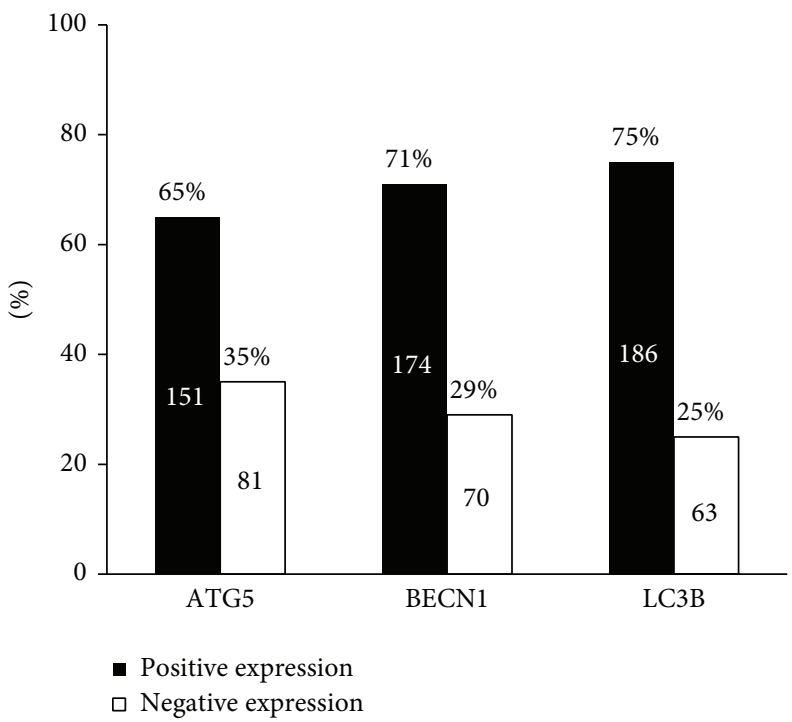

FIGURE 2: Expression levels of autophagy-related proteins were dichotomized into positive and negative categories based on the intensity and percentage of staining. Missing data for ATG5 $(n=31)$, BECN1 $(n=19)$, and LC3B $(n=14)$. 
TABLE 2: Relationships among clinicopathological factors and the expression patterns of autophagy-related proteins.

\begin{tabular}{|c|c|c|c|c|c|c|}
\hline & \multicolumn{2}{|c|}{ ATG5 } & \multicolumn{2}{|c|}{ BECN1 } & \multicolumn{2}{|c|}{ LC3B } \\
\hline & Negative, $n(\%)$ & Positive, $n(\%)$ & Negative, $n(\%)$ & Positive, $n(\%)$ & Negative, $n(\%)$ & Positive, $n(\%)$ \\
\hline \multicolumn{7}{|l|}{ T stage } \\
\hline $1-2$ & $20(39.2)$ & $31(66.7)$ & $14(28.0)$ & $36(72.0)$ & $8(16.3)$ & $41(83.7)$ \\
\hline 3 & $46(33.8)$ & $90(66.2)$ & $46(30.9)$ & $103(69.1)$ & 43 (27.9) & $111(72.1)$ \\
\hline 4 & $15(33.3)$ & $30(33.7)$ & $10(22.2)$ & $35(77.8)$ & $12(26.1)$ & $34(73.9)$ \\
\hline$P$ & & 0.578 & & 0.444 & & 0.152 \\
\hline \multicolumn{7}{|l|}{$\mathrm{N}$ stage } \\
\hline 0 & $38(38.0)$ & $62(62.0)$ & $35(34.0)$ & $68(66.0)$ & $28(25.9)$ & $80(74.1)$ \\
\hline 1 & $25(35.2)$ & $46(64.8)$ & $23(29.9)$ & $54(70.1)$ & $21(27.6)$ & $55(72.4)$ \\
\hline 2 & $18(29.5)$ & $43(70.5)$ & $12(18.8)$ & $52(81.3)$ & $14(21.5)$ & $51(78.5)$ \\
\hline$P$ & & 0.290 & & 0.044 & & 0.695 \\
\hline \multicolumn{7}{|l|}{ Distant metastasis } \\
\hline No & $68(38.2)$ & $110(61.8)$ & $57(30.0)$ & $133(70.0)$ & $49(25.0)$ & $147(75.0)$ \\
\hline Yes & $13(24.1)$ & $41(75.9)$ & $13(24.1)$ & $41(75.9)$ & $14(26.4)$ & $39(73.6)$ \\
\hline$P$ & & 0.056 & & 0.396 & & 0.833 \\
\hline \multicolumn{7}{|l|}{ Stage } \\
\hline I & $14(37.8)$ & $23(62.2)$ & $11(30.6)$ & $25(69.4)$ & $6(16.7)$ & $30(83.3)$ \\
\hline II & $22(40.7)$ & $32(59.3)$ & $23(39.0)$ & $36(61.0)$ & $20(31.3)$ & $44(68.8)$ \\
\hline III & $32(36.8)$ & $55(63.2)$ & $23(24.2)$ & $72(75.8)$ & $23(24.0)$ & $73(76.0)$ \\
\hline IV & $13(24.1)$ & $41(75.9)$ & $13(24.1)$ & $41(75.9)$ & $14(26.4)$ & $39(73.6)$ \\
\hline$P$ & & 0.104 & & 0.122 & & 0.761 \\
\hline \multicolumn{7}{|l|}{ Histology } \\
\hline Tubular & $72(35.0)$ & $134(63.0)$ & $60(27.8)$ & $156(72.2)$ & $55(25.0)$ & $165(75.0)$ \\
\hline Others $^{\dagger}$ & $9(34.6)$ & $17(65.4)$ & $10(35.7)$ & $18(64.3)$ & $8(27.6)$ & $21(72.4)$ \\
\hline$P$ & & 0.976 & & 0.382 & & 0.763 \\
\hline \multicolumn{7}{|c|}{ Lymphovascular invasion } \\
\hline Negative & $33(37.9)$ & $54(62.1)$ & $30(33.7)$ & $59(66.3)$ & $24(26.1)$ & $68(73.9)$ \\
\hline Positive & $48(33.1)$ & $97(66.9)$ & $40(25.8)$ & $115(74.2)$ & $39(24.8)$ & $118(75.2)$ \\
\hline$P$ & & 0.455 & & 0.189 & & 0.827 \\
\hline \multicolumn{7}{|l|}{ Histological grade } \\
\hline Well/moderately & $66(33.5)$ & $131(66.5)$ & $53(26.0)$ & $151(74.0)$ & $49(23.4)$ & $160(76.6)$ \\
\hline Poorly & $15(42.9)$ & $20(57.1)$ & $17(42.5)$ & $23(57.5)$ & $14(35.0)$ & $26(65.0)$ \\
\hline$P$ & & 0.285 & & 0.035 & & 0.124 \\
\hline
\end{tabular}

${ }^{*}$ Statistically significant $(P<0.05)$.

${ }^{\dagger}$ Mucinous carcinoma $(n=28)$, signet-ring-cell carcinoma $(n=2)$.

$(P<0.001)$. Negative LC3B expression was found to be significantly associated with a poor outcome $(P=0.019$; Figure 3(c)). However, the expression levels of BECN1 and ATG5 were not significantly correlated with overall survival (Figures 3(a) and 3(b)). Multivariate analysis was performed to identify relationships between the previously mentioned factors and prognosis. Curative resection status $(P<0.001)$, lymph node involvement $(P<0.001)$, and distant metastasis $(P=0.008)$ were significant poor prognostic factors. In addition, negative LC3B expression was found to be an independent indicator of poor prognosis (hazard ratio, 0.518; 95\% CI, 0.319-0.841; $P=0.008$; Table 4 and Figure 3(c)).

3.4. Changes in the Expression of Autophagy-Related Proteins and Their Clinical Significance. The number of autophagyrelated proteins with positive expression ranged from 0 to 3 with a mean of $2.1 \pm 0.7$. The available 217 cases were classified into two groups according to the number of proteins that were expressed. In 29 cases, no autophagy-related protein was expressed, and these cases were defined as the negative autophagy type. The other 188 cases showed the expression of 1 to 3 proteins and were designated as the positive autophagy type. When the clinicopathological characteristics were analyzed according to these autophagy types, the negative autophagy type was found to be significantly associated with poor histological differentiation $(P=0.014)$. In the univariate analysis, patients with the negative autophagy type had a significantly worse prognosis than those with the positive autophagy type; the median overall survival time was 43.7 months (95\% CI, 10.4-77.0) for negative-autophagytype patients and had not yet been reached for positiveautophagy-type patients $(P=0.002)$. Multivariate analysis 
TABLE 3: Relationships among the expression patterns of autophagyrelated proteins.

\begin{tabular}{lcccc}
\hline & \multicolumn{2}{c}{ LC3B } & \multicolumn{2}{c}{ BECN1 } \\
& $\begin{array}{c}\text { Negative } \\
(\%)\end{array}$ & $\begin{array}{c}\text { Positive } \\
(\%)\end{array}$ & $\begin{array}{c}\text { Negative } \\
(\%)\end{array}$ & $\begin{array}{c}\text { Positive } \\
(\%)\end{array}$ \\
\hline ATG5 & & & & \\
Negative (\%) & $37(47.4)$ & $41(52.6)$ & $43(53.8)$ & $37(46.3)$ \\
Positive (\%) & $17(11.6)$ & $130(88.4)$ & $15(10.5)$ & $128(89.5)$ \\
$P$ & & $<0.001$ & & $<0.001$ \\
Beclin-1 & & & \\
Negative (\%) & $37(56.9)$ & $28(43.1)$ & \\
Positive (\%) & $20(12.0)$ & $147(88.0)$ & & \\
$P$ & & $<0.001$ & & \\
\hline
\end{tabular}

TABLE 4: Multivariate analysis of the clinicopathological parameters and three autophagy-related proteins by overall survival rate using the Cox proportional hazards model.

\begin{tabular}{lccc}
\hline Characteristics & Hazard ratio & $95 \%$ CI & $P$ \\
\hline $\begin{array}{l}\text { LC3B expression } \\
\text { (positive versus negative) }\end{array}$ & 0.518 & $0.319-0.841$ & 0.008 \\
$\begin{array}{l}\text { Lymph node involvement } \\
\text { (yes versus no) }\end{array}$ & 4.349 & $2.275-8.313$ & $<0.001$ \\
$\begin{array}{l}\text { Curative resection } \\
\text { (no versus yes) }\end{array}$ & 4.322 & $2.177-8.582$ & $<0.001$ \\
$\begin{array}{l}\text { Distant metastasis } \\
\text { (yes versus no) }\end{array}$ & 2.504 & $1.273-4.924$ & 0.008 \\
\hline
\end{tabular}

Data calculated using the Cox proportional hazards model. CI: confidence interval.

showed that curative resection status $(P<0.001)$, lymph node involvement $(P=0.019)$, and distant metastasis $(P=0.007)$ were strong predictive factors. In addition, the negative autophagy type was an independent prognostic factor (hazard ratio, 0.432 ; 95\% CI, $0.241-0.774 ; P=0.005$; see Table 5 and Figure 4).

\section{Discussion}

Emerging evidence has shown that tumorigenesis and the progression of human cancers are affected by disturbances in the molecular machinery regulating autophagy $[1,3]$. However, there are a limited number of studies of autophagy markers in CRC. The results of the present study suggest that the negative expression of various autophagy-related proteins is associated with poor clinical outcome in CRC, and the prognostic impact of these proteins seemed to be independent of well-known clinicopathological parameters. In contrast to some human cancers, including ovarian, breast, and prostate cancers, the major gastrointestinal cancers (esophageal, stomach, and colorectal cancers) exhibit high levels of autophagy activity $[19,23]$. Ahn et al. reported that BECN1 protein expression, assessed by immunohistochemistry, was detected in 98 (95\%) of 103 samples of colorectal adenocarcinoma and in $50(83 \%)$ of 60 samples of gastric adenocarcinoma [23]. In this study, there was no significant
TABLE 5: Predictive factors of survival by multivariate analysis using autophagy score (Cox proportional hazards model).

\begin{tabular}{lccc}
\hline Characteristics & Hazard ratio & $95 \%$ CI & $P$ \\
\hline $\begin{array}{l}\text { Autophagy score } \\
(1-3 \text { versus 0) }\end{array}$ & 0.432 & $0.241-0.774$ & 0.005 \\
$\begin{array}{l}\text { Lymph node } \\
\text { involvement } \\
\text { (yes versus no) }\end{array}$ & 2.734 & $1.184-6.316$ & 0.019 \\
$\begin{array}{l}\text { Curative resection } \\
\text { (no versus yes) }\end{array}$ & 3.846 & $1.950-7.584$ & $<0.001$ \\
$\begin{array}{l}\text { Distant metastasis } \\
\text { (yes versus no) }\end{array}$ & 2.528 & $1.282-4.987$ & 0.007 \\
\hline
\end{tabular}

Data calculated using the Cox proportional hazards model. CI: confidence interval.

association between BECN1 expression and the clinicopathological characteristics. Other previous studies have also found that autophagy-related proteins were upregulated in colon cancer, as assessed by an increase in LC3 or BECN1 expression in a significant proportion of primary tumors [16, 24-26].

The expression of autophagy-related proteins (particularly LC3 and BECN1) has been reported to be a prognostic factor in various human cancers, but the results are conflicting. Giatromanolaki et al. demonstrated that the LC3A and BECN1 proteins are highly expressed in breast, lung, endometrial, urothelial, and prostate tumors, and the expression of these proteins was significantly associated with tumor aggressiveness and poor prognosis [27]. The authors classified LC3A immunoreactivity into three types according to the staining pattern in breast carcinoma, namely, the diffuse cytoplasmic, perinuclear, and "stone-like" intracellular structure (SLS) types, each of which had a distinct prognostic relevance [28]. In colorectal adenocarcinoma, perinuclear LC3A expression, indicative of a basal level of autophagic function, has been shown to be an independent marker of good prognosis, but high SLS counts, presumably reflecting an excessive autophagic response, were associated with tumor hypoxia, metastases, and poor prognosis [29]. LC3A and B proteins are generally believed to have similar functions in the initiation and formation of autophagosomes [27]. In our study, negative LC3B expression was found to be significantly associated with poor outcomes. However, Zheng et al. reported that LC3B expression in the peripheral area of colorectal cancer tissues was significantly correlated with several clinicopathological parameters [30]. In addition, Guo et al. demonstrated that patients with low LC3 expression had a higher objective response rate among advanced colorectal cancer patients treated with cetuximab-containing chemotherapy [31]. These conflicting results could be due to the variable prognostic value of LC3, which depends on the intrinsic properties of the tumor, the stage, and the treatment regimen.

The present study showed that other key autophagyrelated proteins, such as BECN1 and ATG5, were not associated with prognosis, although positive BECN1 expression was significantly correlated with good histological differentiation. BECN1 expression has been widely studied to determine its association with the prognosis of CRC. However, several 


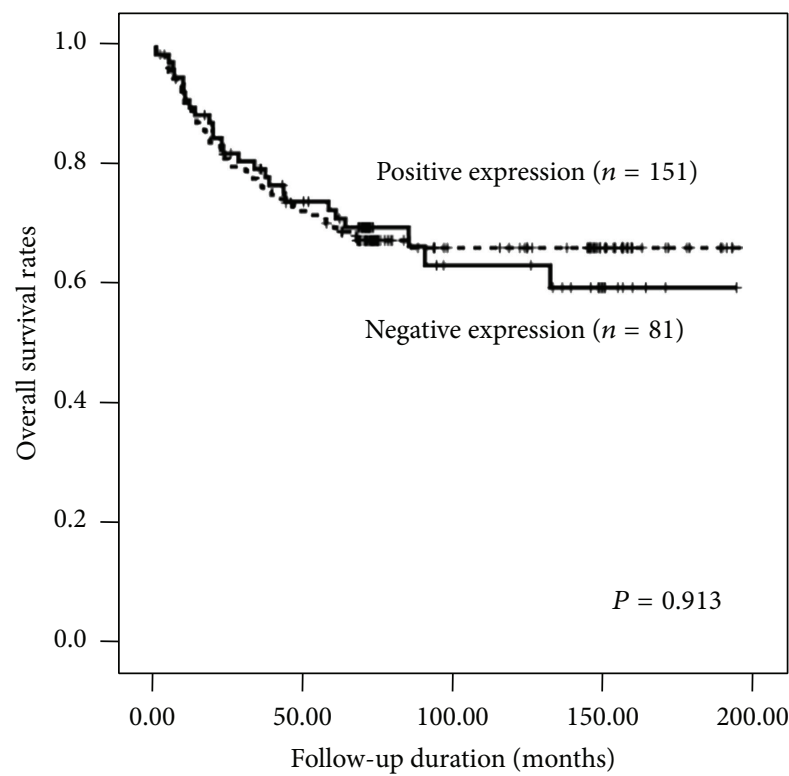

(a)

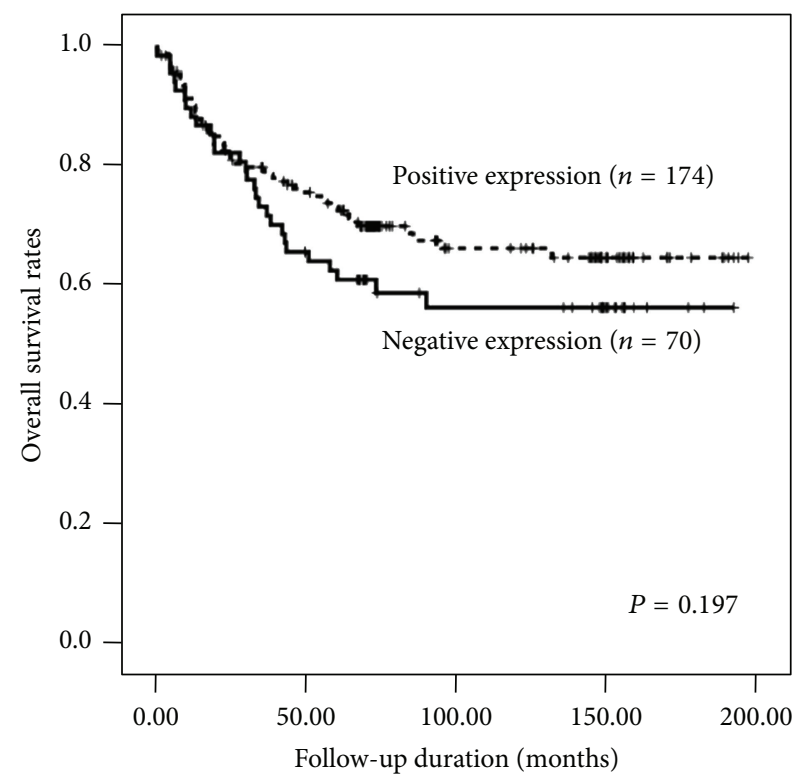

(b)

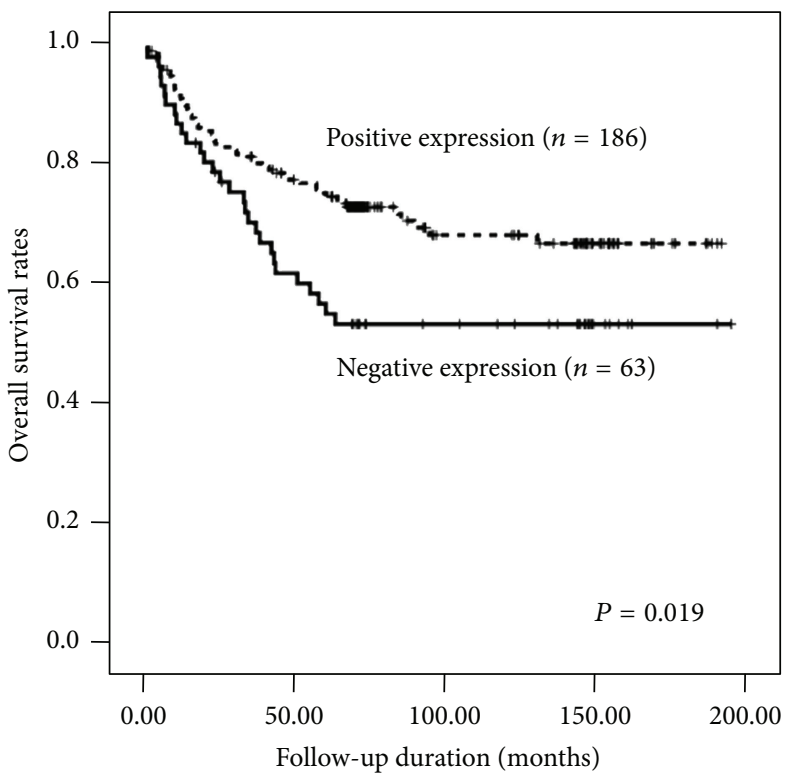

(c)

FIGURE 3: Survival curves using the Kaplan-Meier method by a log-rank test. (a) ATG5 expression. (b) BECN1 expression. (c) LC3B expression.

studies have yielded conflicting results. Li et al. reported that high BECN1 expression was associated with favorable outcomes in resected stage IIIB colon cancers treated with 5-FUbased adjuvant chemotherapy after surgery [24]. Recently, Koukourakis et al. classified the BECN1 expression patterns in colorectal cancer patients treated with surgery alone into four categories, combining the extent and intensity of staining [25]. The four groups were the normal-like, limited overexpression, extensive overexpression, and underexpression groups. In this study, the underexpression of BECN1 was correlated with poor prognosis, whereas the extensive overexpression of BECN1 was associated with the tumor HIF$1 \alpha$ level and aggressive clinical behavior. Other studies have also linked BECN1 overexpression with reduced survival in colon cancer patients treated with adjuvant 5-FU [26] and lower BECN1 expression with a longer median progressionfree survival in patients with advanced colorectal cancer treated with cetuximab-containing chemotherapy [31]. As for LC3 expression, the heterogeneity due to the mix of colon and rectal cancers as well as the variation in the stage from stage I to IV complicates the interpretation of the prognostic value of BECN1 expression in colorectal cancers [24].

Data regarding the role of autophagy-related proteins other than LC3 and BECN1 are still lacking. A recent study by Cho et al. demonstrated that ATG5 expression was found in the tumors of approximately 80\% (102/124) of colorectal 


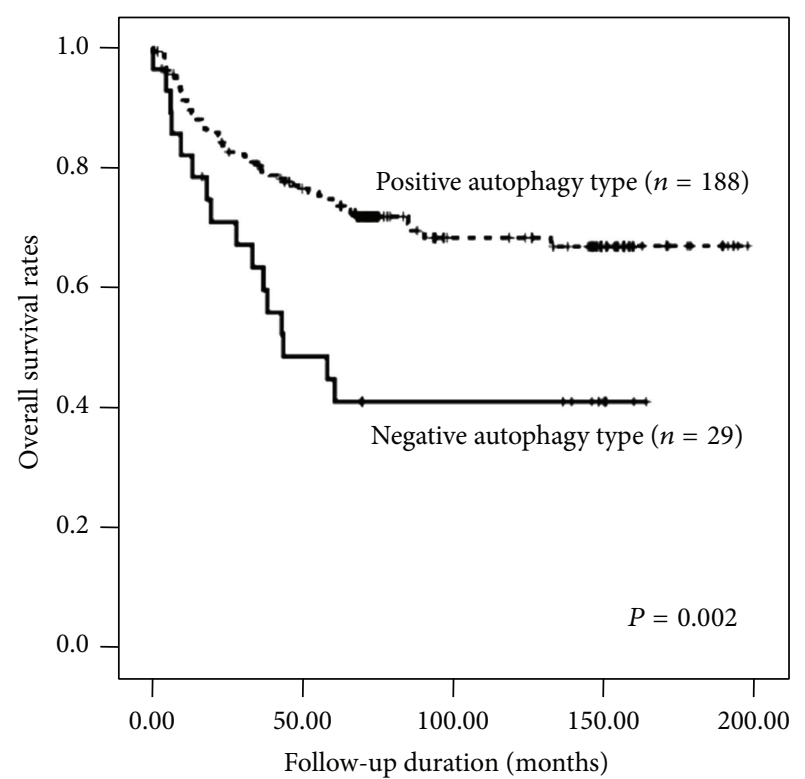

Figure 4: Survival curves using the Kaplan-Meier method by a logrank test for autophagy type in all cases.

cancer patients, and high protein expression correlated with lymphovascular invasion, which is a risk factor for recurrence and poor survival outcome [32]. However, there was no correlation between ATG5 expression and overall survival or disease-free survival. Another study reported that ATG10, one of the E2-like conjugation enzymes for ATG12-ATG5 conjugation, was highly increased in colorectal cancer tissues, and increased protein expression was associated with lymphovascular invasion and lymph node metastasis [33].

Because the associations among the expression levels of the autophagy-related proteins (ATG5, BECN1, and LC3B) were strong and significant, consistent with their known roles in regulating autophagy activation, we divided the patients into two groups according to the number of autophagyrelated protein expression changes. The positive autophagy type was associated with good histological differentiation and a favorable clinical outcome. Although the curative resection status was the strongest prognostic factor in the multivariate analysis, the change in autophagy-related protein expression was also an independent prognostic factor. Taken together, these findings suggest that the number of autophagy-related protein changes is significantly associated with tumor progression. Recently, the roles of autophagy in colorectal cancer development and treatment have been investigated both in vitro and in vivo. The transfection of the low BECN1 gene-expressing colon cancer cells with the $B E C N 1$ gene inhibited cell growth, and cell cycle analysis revealed G1 arrest, indicating that BECN1 plays an important role in the proliferation of colorectal cancer cells [34]. In contrast, autophagy also may play an important role in the survival of colorectal cancer cells, suggesting that the role of autophagy in colorectal cancer may be complex [16].

Sex significantly influences several clinicopathological characteristics of CRC, including incidence and mortality rates, survival chemotherapeutic response, and certain molecular characteristics [35-37]. Emerging evidence indicates that estrogens and/or progestins have a protective effect against colorectal carcinogenesis [36]. This protective effect of estrogens is mediated by interaction with estrogen receptor $\beta$ $(\operatorname{ER} \beta)$, microsatellite status, and progressive hypermethylation of the CpG islands of the promoter region of the ER [37]. In the present study, there were no differences in autophagyrelated proteins expression according to sex, and the distribution of conventional clinicopathological and prognostic factors did not differ between female and male CRC. However, recent in vitro study has demonstrated that 2-methoxyestradiol, which derives from the NADPH-dependent cytochrome P450 metabolism of $17 \beta$-estradiol, is able to induce apoptosis as well as autophagy in colon carcinoma-derived cell lines [38]. This result suggests that autophagy could play a role for gender differences in CRC. Further research for the exact molecular mechanisms is necessary.

Studies regarding autophagy in cancer tissue sections are limited by the difficulty in performing dynamic assays, which are necessary for monitoring autophagy. Although counting autophagic vesicles by electron microscopy is the standard approach for detecting autophagy, this method is not readily accessible and is not capable of evaluating entire tumor samples [39]. Immunohistochemical staining is a useful method for monitoring autophagic activity in surgically resected cancer specimens, and the advantages of this method include the ability to analyze changes in the expression levels of a number of autophagy-related proteins. The p62/sequestosome 1 (SQSTM1), a cytosolic adaptor protein, facilitates the autophagic degradation of ubiquitinated protein aggregates in lysosomes [40]. The impaired turnover of p62 due to defective or impaired autophagy is associated with the accumulation of p62 [41]. p62 can also be used as a protein marker of autophagy [39]. In a future study, adding p62 would be helpful to improve prognostic role of autophagy-related proteins. This study is also limited by the small number of patients, and the results cannot be considered representative of all colorectal cancer patients. To determine the role of changes in autophagy-related protein expression levels as prognostic factors, further validation and standardization of the immunohistochemical assay are required, in addition to a larger number of colorectal cancer patients.

In conclusion, we found that negative LC3B expression and absence of autophagy-related proteins expression were independently associated with poor survival in patients with CRC. Because the expression levels of autophagy-related proteins showed no correlation with well-known prognostic markers, these proteins have potential uses as novel prognostic markers in patients with colorectal cancer.

\section{Conflict of Interests}

All authors have no conflict of interests to declare.

\section{Authors' Contribution}

Y.-S. Cho and Y. H. Ko contributed equally to this study. 


\section{Acknowledgments}

This study was supported by a grant of the Institute of Clinical Medicine Research, Uijeongbu St. Mary's Hospital, The Catholic University of Korea (Y.-S. Cho \& Y. H. Ko) and a grant of Basic Science Research Program through the National Research Foundation of Korea funded by the Ministry of Education, Science and Technology (NRF-20100023295) (Y.-S. Cho).

\section{References}

[1] B. Levine and G. Kroemer, "Autophagy in the pathogenesis of disease," Cell, vol. 132, no. 1, pp. 27-42, 2008.

[2] N. Mizushima, B. Levine, A. M. Cuervo, and D. J. Klionsky, "Autophagy fights disease through cellular self-digestion," Nature, vol. 451, no. 7182, pp. 1069-1075, 2008.

[3] R. Mathew, V. Karantza-Wadsworth, and E. White, "Role of autophagy in cancer," Nature Reviews Cancer, vol. 7, no. 12, pp. 961-967, 2007.

[4] X. Qu, J. Yu, G. Bhagat et al., "Promotion of tumorigenesis by heterozygous disruption of the beclin 1 autophagy gene," Journal of Clinical Investigation, vol. 112, no. 12, pp. 1809-1820, 2003.

[5] Z. Yue, S. Jin, C. Yang, A. J. Levine, and N. Heintz, "Beclin 1, an autophagy gene essential for early embryonic development, is a haploinsufficient tumor suppressor," Proceedings of the National Academy of Sciences of the United States of America, vol. 100, no. 25, pp. 15077-15082, 2003.

[6] K. N. Dalby, I. Tekedereli, G. Lopez-Berestein, and B. Ozpolat, "Targeting the prodeath and prosurvival functions of autophagy as novel therapeutic strategies in cancer," Autophagy, vol. 6, no. 3, pp. 322-329, 2010.

[7] S. Roy and J. Debnath, "Autophagy and tumorigenesis," Seminars in immunopathology, vol. 32, no. 4, pp. 383-396, 2010.

[8] M. P. Tschan and H.-U. Simon, "The role of autophagy in anticancer therapy: promises and uncertainties," Journal of Internal Medicine, vol. 268, no. 5, pp. 410-418, 2010.

[9] M. J. Abedin, D. Wang, M. A. McDonnell, U. Lehmann, and A. Kelekar, "Autophagy delays apoptotic death in breast cancer cells following DNA damage," Cell Death and Differentiation, vol. 14, no. 3, pp. 500-510, 2007.

[10] J. S. Carew, S. T. Nawrocki, C. N. Kahue et al., "Targeting autophagy augments the anticancer activity of the histone deacetylase inhibitor SAHAto overcome Bcr-Abl-mediated drug resistance," Blood, vol. 110, no. 1, pp. 313-322, 2007.

[11] T. Nishikawa, N. H. Tsuno, Y. Okaji et al., "Inhibition of autophagy potentiates sulforaphane-induced apoptosis in human colon cancer cells," Annals of Surgical Oncology, vol. 17, no. 2, pp. 592-602, 2010.

[12] P. Pisani, D. M. Parkin, F. Bray, and J. Ferlay, "Estimates of the worldwide mortality from 25 cancers in 1990," International Journal of Cancer, vol. 83, no. 1, pp. 18-29, 1999.

[13] T. Watanabe, T.-T. Wu, P. J. Catalano et al., "Molecular predictors of survival after adjuvant chemotherapy for colon cancer," The New England Journal of Medicine, vol. 344, no. 16, pp. 1196-1206, 2001.

[14] C. B. Diep, L. Thorstensen, G. I. Meling, E. Skovlund, T. O. Rognum, and R. A. Lothe, "Genetic tumor markers with prognostic impact in Dukes' stages B and C colorectal cancer patients," Journal of Clinical Oncology, vol. 21, no. 5, pp. 820829, 2003.
[15] D. Arango, P. Laiho, A. Kokko et al., "Gene-expression profiling predicts recurrence in Dukes' C colorectal cancer," Gastroenterology, vol. 129, no. 3, pp. 874-884, 2005.

[16] K. Sato, K. Tsuchihara, S. Fujii et al., "Autophagy is activated in colorectal cancer cells and contributes to the tolerance to nutrient deprivation," Cancer Research, vol. 67, no. 20, pp. $9677-$ 9684, 2007.

[17] H. Nakatogawa, K. Suzuki, Y. Kamada, and Y. Ohsumi, "Dynamics and diversity in autophagy mechanisms: lessons from yeast," Nature Reviews Molecular Cell Biology, vol. 10, no. 7, pp. 458-467, 2009.

[18] X. H. Liang, S. Jackson, M. Seaman et al., "Induction of autophagy and inhibition of tumorigenesis by beclin 1," Nature, vol. 402, no. 6762, pp. 672-676, 1999.

[19] A. Yoshioka, H. Miyata, Y. Doki et al., "LC3, an autophagosome marker, is highly expressed in gastrointestinal cancers," International Journal of Oncology, vol. 33, no. 3, pp. 461-468, 2008.

[20] D. J. Klionsky, "Autophagy: from phenomenology to molecular understanding in less than a decade," Nature Reviews Molecular Cell Biology, vol. 8, no. 11, pp. 931-937, 2007.

[21] J. Kononen, L. Bubendorf, A. Kallioniemi et al., "Tissue microarrays for high-throughput molecular profiling of tumor specimens," Nature Medicine, vol. 4, no. 7, pp. 844-847, 1998.

[22] Y. H. Ko, Y. S. Cho, H. S. Won et al., "Prognostic significance of autophagy-related protein expression in resected pancreatic ductal adenocarcinoma," Pancreas, vol. 42, no. 5, pp. 829-835, 2013.

[23] C. H. Ahn, E. G. Jeong, and J. W. Lee, "Expression of beclin-1, an autophagy-related protein, in gastric and colorectal cancers," Acta Pathologica, Microbiologica et Immunologica Scandinavica, vol. 115, no. 5, pp. 1344-1349, 2007.

[24] B.-X. Li, C.-Y. Li, R.-Q. Peng et al., "The expression of beclin 1 is associated with favorable prognosis in stage IIIB colon cancers," Autophagy, vol. 5, no. 3, pp. 303-306, 2009.

[25] M. I. Koukourakis, A. Giatromanolaki, E. Sivridis, M. Pitiakoudis, K. C. Gatter, and A. L. Harris, "Beclin 1 over- and underexpression in colorectal cancer: distinct patterns relate to prognosis and tumour hypoxia," British Journal of Cancer, vol. 103, no. 8, pp. 1209-1214, 2010.

[26] J. Myung Park, S. Huang, T. T. Wu, N. R. Foster, and F. A. Sinicrope, "Prognostic impact of Beclin 1, p62/sequestosome 1 and LC3 protein expression in colon carcinomas from patients receiving 5-fluorouracil as adjuvant chemotherapy," Cancer Biology \& Therapy, vol. 14, no. 2, pp. 100-107, 2013.

[27] A. Giatromanolaki, E. Sivridis, S. Mendrinos, A. V. Koutsopoulos, and M. I. Koukourakis, "Autophagy proteins in prostate cancer: relation with anaerobic metabolism and Gleason score," Urologic Oncology, vol. 32, no. 1, pp. 39.e11-39.e18, 2013.

[28] E. Sivridis, M. I. Koukourakis, C. E. Zois et al., "LC3A-positive light microscopy detected patterns of autophagy and prognosis in operable breast carcinomas," American Journal of Pathology, vol. 176, no. 5, pp. 2477-2489, 2010.

[29] A. Giatromanolaki, M. I. Koukourakis, A. L. Harris, A. Polychronidis, K. C. Gatter, and E. Sivridis, "Prognostic relevance of light chain 3 (LC3A) autophagy patterns in colorectal adenocarcinomas," Journal of Clinical Pathology, vol. 63, no. 10, pp. 867-872, 2010.

[30] H. Y. Zheng, X. Y. Zhang, X. F. Wang, and B. C. Sun, "Autophagy enhances the aggressiveness of human colorectal cancer cells and their ability to adapt to apoptotic stimulus," Cancer Biology \& Medicine, vol. 9, no. 2, pp. 105-110, 2012. 
[31] G.-F. Guo, W.-Q. Jiang, B. Zhang et al., "Autophagy-related proteins Beclin-1 and LC3 predict cetuximab efficacy in advanced colorectal cancer," World Journal of Gastroenterology, vol. 17, no. 43, pp. 4779-4786, 2011.

[32] D. H. Cho, Y. K. Jo, S. C. Kim, I. J. Park, and J. C. Kim, "Downregulated expression of ATG5 in colorectal cancer," Anticancer Research, vol. 32, no. 9, pp. 4091-4096, 2012.

[33] Y. K. Jo, S. C. Kim, I. J. Park et al., "Increased expression of ATG10 in colorectal cancer is associated with lymphovascular invasion and lymph node metastasis," PLoS ONE, vol. 7, no. 12, Article ID e52705, 2012.

[34] K. Koneri, T. Goi, Y. Hirono, K. Katayama, and A. Yamaguchi, "Beclin 1 gene inhibits tumor growth in colon cancer cell lines," Anticancer Research, vol. 27, no. 3, pp. 1453-1457, 2007.

[35] M. L. Slattery, J. D. Potter, K. Curtin et al., "Estrogens reduce and withdrawal of estrogens increase risk of microsatellite instability-positive colon cancer," Cancer Research, vol. 61, no. 1, pp. 126-130, 2001.

[36] S. Wangefjord, J. Manjer, A. Gaber, B. Nodin, J. Eberhard, and K. Jirstrom, "Cyclin D1 expression in colorectal cancer is a favorable prognostic factor in men but not in women in a prospective, population-based cohort study," Biology of Sex Differences, vol. 2, article 10, 2011.

[37] J. H. Koo and R. W. L. Leong, "Sex differences in epidemiological, clinical and pathological characteristics of colorectal cancer," Journal of Gastroenterology and Hepatology, vol. 25, no. 1, pp. 33-42, 2010.

[38] P. Lista, E. Straface, S. Brunelleschi, F. Franconi, and W. Malorni, "On the role of autophagy in human diseases: a gender perspective," Journal of Cellular and Molecular Medicine, vol. 15, no. 7, pp. 1443-1457, 2011.

[39] N. Mizushima, T. Yoshimori, and B. Levine, "Methods in mammalian autophagy research," Cell, vol. 140, no. 3, pp. 313326, 2010.

[40] S. Pankiv, T. H. Clausen, T. Lamark et al., "p62/SQSTM1 binds directly to Atg8/LC3 to facilitate degradation of ubiquitinated protein aggregates by autophagy*," The Journal of Biological Chemistry, vol. 282, no. 33, pp. 24131-24145, 2007.

[41] R. Mathew, C. M. Karp, B. Beaudoin et al., "Autophagy suppresses tumorigenesis through elimination of p62," Cell, vol.137, no. 6, pp. 1062-1075, 2009. 


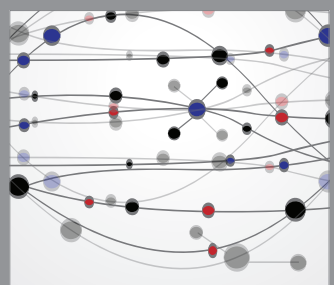

The Scientific World Journal
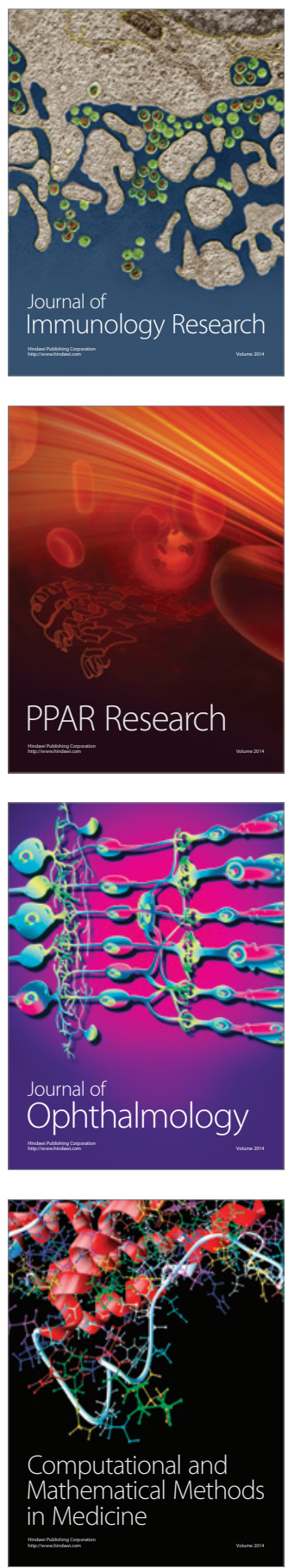

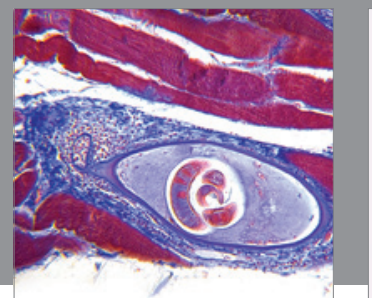

Gastroenterology

Research and Practice
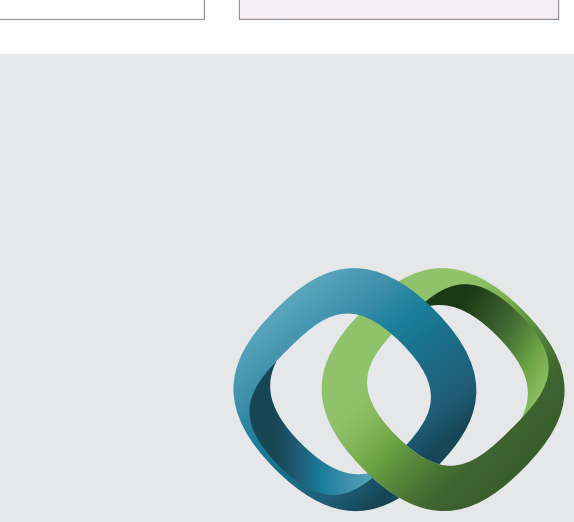

\section{Hindawi}

Submit your manuscripts at

http://www.hindawi.com
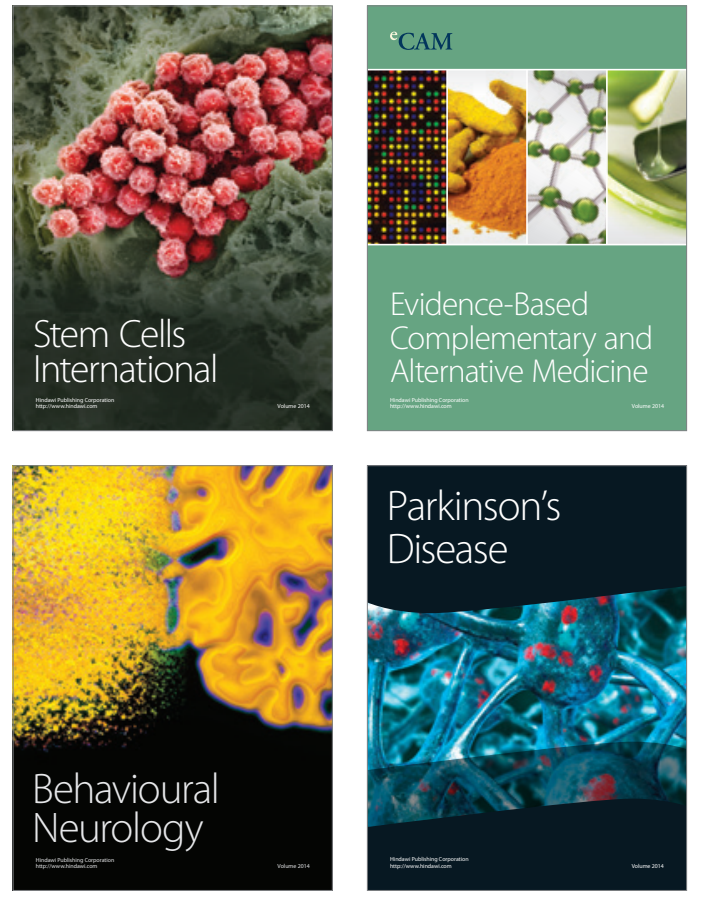
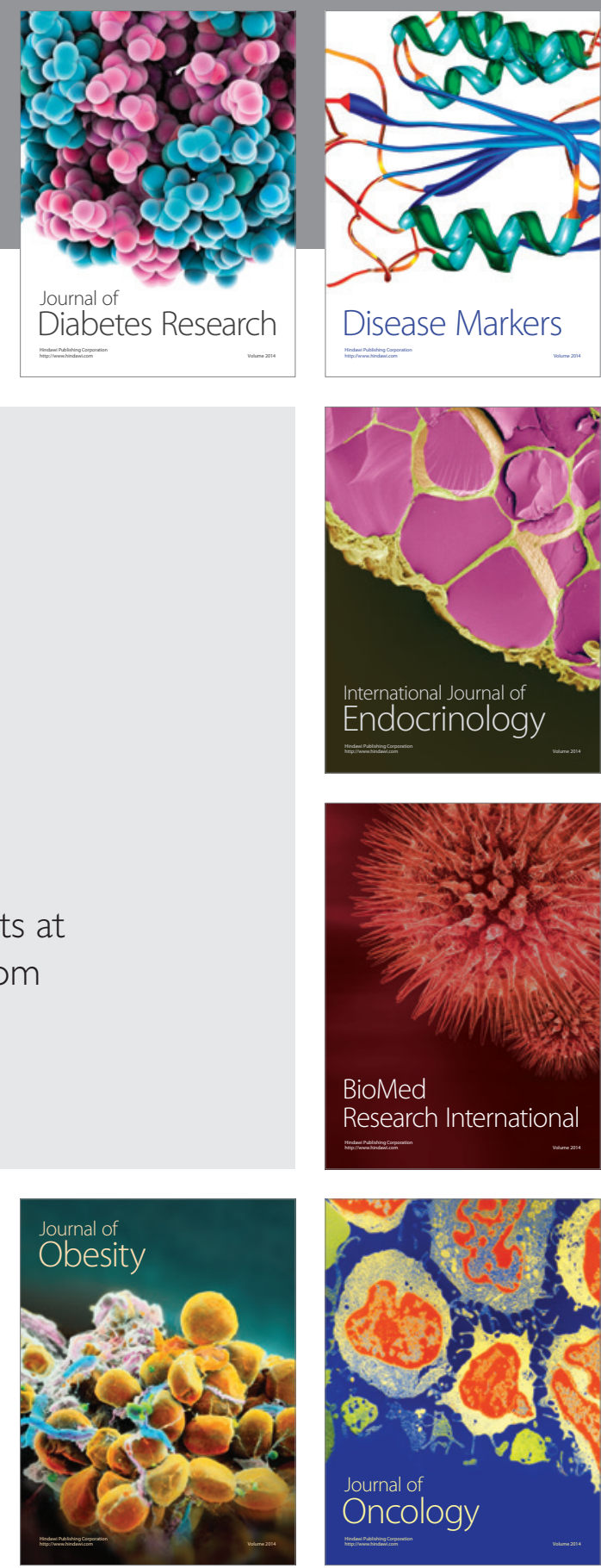

Disease Markers
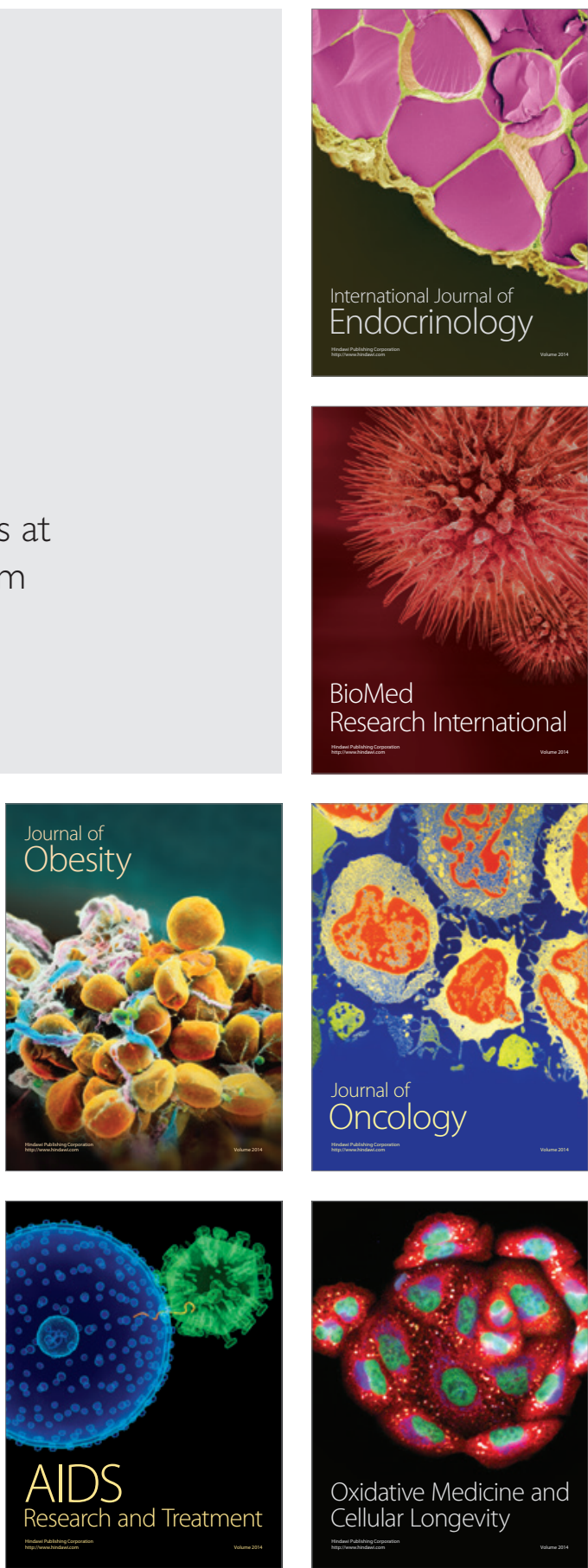